\title{
3. Zusammenarbeit mit Osteuropa und der GUS
}

\section{Tanja Guggenbühl}

\section{OpenEdition}

\section{Journals}

Electronic version

URL: http://journals.openedition.org/sjep/108

DOI: $10.4000 /$ sjep. 108

ISSN: 1663-9677

\section{Publisher}

Institut de hautes études internationales et du développement

\section{Printed version}

Date of publication: 1 avril 2007

Number of pages: $35-42$

ISBN: 978-2-88247-067-6

ISSN: $1660-5926$

\section{Electronic reference}

Tanja Guggenbühl, « 3. Zusammenarbeit mit Osteuropa und der GUS », Schweizerisches Jahrbuch für Entwicklungspolitik [Online], 26-1 | 2007, Online erschienen am: 29 März 2010, abgerufen am 08 September 2020. URL : http://journals.openedition.org/sjep/108 ; DOI : https://doi.org/10.4000/sjep 108

(c) The Graduate Institute 


\section{Zusammenarbeit mit Osteuropa und der GUS*}

D IE TRANSITIONSHILFE der Schweiz für Osteuropa und die GUS hat eine neue Gesetzesgrundlage. Das Bundesgesetz über die Zusammenarbeit mit den Staaten Osteuropas wurde vom Schweizer Volk am 26. November 2006 mit 53,4 Prozent Jastimmen nach einem Referendum angenommen.

Das Gesetz umfasst auch die rechtliche Grundlage für den Beitrag der Schweiz zur Verringerung der wirtschaftlichen und sozialen Ungleichheiten in der erweiterten Europäischen Union (EU). Es bestätigt somit die politische Absichtserklärung, die der Bundesrat in Form eines Memorandum of Understanding mit Brüssel im Februar 2006 unterzeichnet hatte. Das Memorandum legt die allgemeinen Modalitäten des Beitrags der Schweiz für die zehn neuen EU-Staaten fest. Der Bundesrat hat hierzu einen Rahmenkredit von einer Milliarde Franken zu Händen der Eidgenössischen Räte genehmigt.

Zum anderen wurde die traditionelle Ostzusammenarbeit der Schweiz im Jahr 2006 fortgesetzt. Der Bundesrat hat dem Parlament einen Antrag für einen vierten Rahmenkredit unterbreitet, dessen Betrag jedoch geringer als bei den drei vorhergehenden Rahmenkrediten ist. Diese Frage wird von den Eidgenössischen Räten im Frühjahr 2007 diskutiert.

\subsection{Neues Bundesgesetz über die Zusammenarbeit mit den Staaten Osteuropas}

Am 26. November 2006 haben die Schweizer Stimmbürger das Bundesgesetz über die Zusammenarbeit mit den Staaten Osteuropas mit 53,4 Prozent Jastimmen angenommen ${ }^{1}$. Die Spaltung ergab sich vor allem zwischen Stadt und Land $^{2}$. Das Tessin hat das Gesetz mit 62,9 Prozent abgelehnt. Im März 2006 hatte das Parlament das Gesetz über die Ostzusammenarbeit mit grosser Mehrheit angenommen ${ }^{3}$, doch machte das von der konservativen Rechten eingebrachte Referendum eine Volksabstimmung erforderlich.

Das neue Gesetz über die Ostzusammenarbeit dient als Rechtsgrundlage für zwei Formen der Schweizer Hilfe: zum einen für den EU-Erweiterungsbeitrag der Schweiz, zum anderen für die Fortsetzung der Transitionshilfe an die ehemals kommunistischen Staaten Osteuropas und Zentralasiens in Ersetzung des Bundesbeschlusses von 1995. Die Geltungsdauer des neuen Gesetzes ist auf zehn Jahre beschränkt.

* Von Tanja Guggenbühl, wissenschaftliche Mitarbeiterin am IUED.

1 Bundesgesetz über die Zusammenarbeit mit den Staaten Osteuropas vom 24. März 2006 (BB1 2006 3529).

2 Die durchschnittliche Annahmequote für die städtischen Gemeinden war 55,9\% gegenüber 43,6\% für die ländlichen Gemeinden. Quelle: Bundesamt für Statistik (BFS), „Abstimmung vom 26. November 2006“, <http://www.bfs.admin.ch>>Politik >Abstimmungen.

3 Das Gesetz wurde vom Nationalrat mit 127 Stimmen (bei 53 Gegenstimmen und 14 Enthaltungen) und vom Ständerat mit 37 Stimmen (bei einer Gegenstimme und 6 Enthaltungen) verabschiedet. 


\subsubsection{Referendum und Kampagnen in Verbindung mit der Volksabstimmung}

Im Juli 2006 ergriffen drei Komitees ein Referendum gegen das Osthilfegesetz Diese Komitees wurden hauptsächlich von der Schweizerischen Volkspartei (SVP), der Aktion für eine unabhängige und neutrale Schweiz (AUNS), den Schweizer Demokraten und der Lega dei Tecinesi unterstützt. Die SVP kündigte in ihrer Argumentation an, sie widersetze sich nicht dem Prinzip der Zahlung einer Milliarde Franken als Kohäsionsbeitrag für die neuen EU-Staaten, sondern lediglich den Parlamentsbeschlüssen betreffend die finanzielle Verwaltung des Beitrags. Wörtlich führte die SVP an: „Das vorliegende Gesetz entspricht einem Freipass für nach oben offene Zahlungen an die EU, ohne dass eine Kompensation im Budget geregelt würde. " ${ }^{5}$ Zum anderen prangerte die SVP die vom Bundesrat beschlossenen Finanzierungsmodalitäten an, die eine Kompensation des Beitrags auch aus dem allgemeinen Bundeshaushalt und nicht nur durch Kürzungen bei den Budgets der beiden betroffenen Departmente vorsehen ${ }^{6}$.

Die Argumente des Bundesrates zugunsten des Osthilfegesetzes betrafen im Wesentlichen den Kohäsionsbeitrag, da die traditionelle Osthilfe von den Initiatoren des Referendums nicht infrage gestellt wurde. Die Hauptargumente waren die Festigung des bilateralen Wegs sowie die politischen und wirtschaftlichen Vorteile für die Schweiz. Das Argument der schweizerischen Solidarität trat hingegen eher in den Hintergrund. Der Bundesrat hob somit den entscheidenden Ausgang der Volksabstimmung für die Konsolidierung des bilateralen Wegs und die Förderung der Interessen der Schweiz in den bilateralen Beziehungen hervor. Er legte auch die politischen Vorteile eines solchen Beitrags dar - insbesondere was Sicherheit, Stabilität und Migrationsverhütung betrifft - wie auch die wirtschaftlichen Vorteile dank der Öffnung der Märkte der neuen EU-Mitgliedsländer. Schliesslich verwies der Bundesrat auf die Mitveranwortung, welche die Schweiz bei der Förderung von Demokratie und Armutsbekämpfung in Europa trage.

Ein Bündnis verschiedener Parteien nahm an der Kampagne für die Annahme des Gesetzes teil, um gegen das Referendum anzugehen. Die Koalition „Die Zusammenarbeit mit Europa kommt allen zugute“ umfasste Vertreter der Sozialdemokratischen Partei, der Freisinnigen, der Liberalen Partei, der Evangelischen Partei und der Grünen. Die Christlich-demokratische Partei führte ihre eigene Kampagne zugunsten des Osthilfegesetzes durch.

Die Wirtschaftsverbände schlossen sich ebenfalls zusammen, um für das Gesetz Kampagne zu machen. economiesuisse führte insbesondere die Vorteile der Ostzusammenarbeit an. Die Schweizer Wirtschaft sei auf gute Beziehungen zum wichtigsten Handelspartner angewiesen. Würde das Volk die Vorlage ablehnen, müsste die Schweiz mit negativen Konsequenzen rechnen. Wörtlich erklärte economiesuisse: „Nimmt die Beziehung Schaden, leiden darunter Schweizer Unternehmen. Sie hätten in den aufstrebenden Märkten einen schweren Stand“"7.

4 Das „Referendumskomitee gegen die Milliardenzahlungen an die EU“, das „Komitee gegen die OstMilliardezahlung“ und das „Komitee gegen die Ost-Milliardezahlung c/o Schweizer Demokraten“.

SVP, ,SVP-Referendum gegen das Osthilfegsetz“, Pressecommuniqué vom 24. März 2006.

6 SVP, „Bezahlen ohne Ende? 2xNein zum Zulagen-Gesetz und den Ost-Milliarden“, Argumentarium, 25. September 2006.

7 economiesuisse, „Ohne Risiko weiter auf dem bewährten Weg. Konstruktive Zusammenarbeit mit Osteuropa“, dossierpolitik Nr. 35.2, 9. Oktober 2006. 
Die Vertreter der Hilfswerke, die ihre Besorgnisse bezüglich Budgetkürzungen bei der öffentlichen Entwicklungshilfe für die Kompensation der Kohäsionsbeiträge zur EU-Erweiterung klar zum Ausdruck gebracht hatten, haben schliesslich für das Gesetz Kampagne gemacht. Der Beschluss des Bundesrates, einen Teil der Milliarde aus dem Bundeshaushalt zu finanzieren, wie auch seine erneute Bekräftigung, die Südhilfe nicht zu belangen, brachten die Hilfswerke dazu, das Bundesgesetz zur Ostzusammenarbeit aktiv zu unterstützen ${ }^{8}$.

Tabelle 3.1: Bundesgesetz über die Zusammenarbeit mit den Staaten Osteuropas

\begin{tabular}{|c|c|}
\hline Kohäsionsbeitrag & Transitionshilfe \\
\hline $\begin{array}{l}\text { Vom Bundesrat vorgeschlagener Rahmenkredit: } \\
1 \text { Milliarde Franken (für } 5 \text { Jahrea) }\end{array}$ & $\begin{array}{l}\text { Vom Bundesrat vorgeschlagener Rahmenkredit: } \\
650 \text { Millionen Franken (für mindestens } 4 \text { Jahre) }\end{array}$ \\
\hline $\begin{array}{l}\text { Empfängerländer: Estland, Lettland, Litauen, Malta, } \\
\text { Polen, Slowakei, Slowenien, Tschechische Republik, } \\
\text { Ungarn und Zypernb }\end{array}$ & 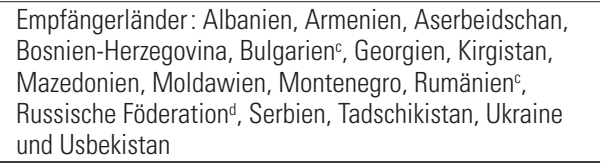 \\
\hline \multicolumn{2}{|c|}{ Die Zahlungen werden sich jedoch über rund zehn Jahre (2007-2016) erstrecken. } \\
\hline \multicolumn{2}{|l|}{ Dabei handelt es sich um die zehn Länder, die d } \\
\hline \multicolumn{2}{|c|}{$\begin{array}{l}\text { c Nach dem EU-Beitritt von Bulgarien und Rumänien im Januar } 2007 \text { werden die Hilfsprogramme der Schweiz in diesen Ländern all- } \\
\text { mählich eingestellt. }\end{array}$} \\
\hline Die Hilfe an die Russische Föderation wird wahrscheinlic & 2008 cingectollt \\
\hline
\end{tabular}

\subsection{Beitrag der Schweiz zum Abbau der wirtschaftlichen und sozialen Disparitäten in der erweiterten Europäischen Union}

Das neue Gesetz über die Ostzusammenarbeit bildet die Rechtsgrundlage für den Schweizer Beitrag zum Abbau der wirtschaftlichen und sozialen Disparitäten in der erweiterten EU. Die Schweiz wird im Rahmen ihres Kohäsionsbeitrags Projekte und Programme in den zehn neuen EU-Mitgliedsstaaten finanzieren (siehe Tabelle 3.1) - mit besonderem Schwerpunkt auf den wirtschaftlich und sozial benachteiligten Regionen.

Hierzu ist zu betonen, dass diese Projekte nicht unter die Entwicklungszusammenarbeit fallen, sondern als Beitrag der Schweiz zur Verringerung der wirtschaftlichen und sozialen Ungleichheiten in der erweiterten EU durchgeführt werden.

\subsubsection{Memorandum of Understanding mit der EU über den Beitrag der Schweiz}

Ein am 27. Februar 2006 unterzeichnetes Memorandum of Understanding 9 (oder politische Absichtserklärung) zwischen der Schweiz und der Europäischen Union legt die Modalitäten des Schweizer Beitrags an die zehn neuen EU-Staaten fest.

Die der Unterzeichnung vorangegangenen Verhandlungen waren langwierig. Bereits im Jahr 2004, als die Schweiz im Begriff war, die zweite Runde der bila-

8 Alliance Sud, „Warum die Hilfswerke Ja sagen zum Osthilfegesetz“, Dossier für die Medienkonferenz vom 20. Oktober 2006, <http://www.alliancesud.ch> > Themen/Kampagnen >Entwicklungspolitik >Spezialdossier EU-Kohäsionsbeitrag > Dossier Medienkonferenz vom 20.10.2006.

9 Memorandum of Unterstanding: <http://www.bundesgesetzost.admin.ch > Dokumentation >Offizielle Texte. Integrationbüro EDA/EVD, „Memorandum of Unterstanding (Erweiterungsbeitrag)“, Pressemitteilung vom 27. Februar 2006. 
teralen Verhandlungen abzuschliessen, hatte sie die Absicht erklärt, einen EUKohäsionsbeitrag von einer Milliarde Franken zu leisten.

[D] Jahrbuch 2005, Nr. 1, Kapitel 3, 3.1. EU-Osterweiterung, S. 37-38.

Bevor das Abkommen unterzeichnet werden konnte, geriet die Ratifizierung der Bilateralen II zwischen der Europäischen Union und der Schweiz wegen Form und Inhalt des Schweizer Kohäsionsbeitrags ins Stocken ${ }^{10}$. Die hauptsächlichen Streitpunkte betrafen die Verteilung und Aufteilung des Schweizer Beitrags, das heisst die Autonomie der Schweiz, ihren Beitrag selbst zu verwalten. Die EU wünschte, dass der schweizerische Beitrag in den europäischen Kohäsionsfonds eingezahlt werde, der auch Spanien, Portugal und Griechenland zugute kommt. Hingegen wollte der Bundesrat einerseits eine bilaterale Hilfe mit jedem Empfängerland einsetzen (und keine Zahlung an den Kohäsionsfonds leisten), und andererseits seine Hilfe nur auf die neuen EU-Staaten beschränken. Die EU akzeptierte die Entscheidung der Schweiz schliesslich Anfang Februar 2006.

Bei der Unterzeichnung des Memorandum of Understanding wies Bundesrätin Micheline Calmy-Rey darauf hin, dass der Beitrag der Schweiz ein Akt der Solidarität sei und gleichzeitig auch die Interessen der Schweiz wahre, da die Integration der neuen Mitgliedsstaaten in die europäischen Institutionen eine Garantie für Stabilität und Wohlstand des gesamten Kontinents darstelle ${ }^{11}$.

Das völkerrechtlich nicht verbindliche Memorandum of Understanding legt den Betrag und die Dauer des Engagements (das auf fünf Jahre beschränkt ist, aber eine Staffelung der Auszahlungen über zehn Jahre vorsieht), ferner die Aufteilung nach Ländern und die Einsatzbereiche fest. Folgende vier Schwerpunktbereiche wurden festgelegt: Sicherheit, Stabilität und Reformen; Infrastruktur und Umwelt; Förderung des Privatsektors ; menschliche und soziale Entwicklung.

Gemäss dem Memorandum of Understanding wird das Hauptempfängerland der Schweizer Hilfe (mit fast 50\% des Beitrags) Polen sein, gefolgt von Ungarn (131 Millionen Franken) und der Tschechischen Republik (110 Millionen Franken). Dieser von der EU vorgeschlagene Verteilschlüssel beruht auf demographischen und sozioökonomischen Kriterien. Die drei genannten Länder, wie auch die anderen neuen EU-Mitglieder werden somit erneut von der Schweiz unterstützt. Ein Teil von ihnen hatte bereits Transitionshilfe erhalten, die in der zweiten Hälfte der 90er Jahre finalisiert worden war.

\subsubsection{Parlamentsdebatten zur Finanzierung des Schweizer Beitrags}

Zuerst hatte der Bundesrat beschlossen, den Kohäsionsbeitrag durch Kompensation in den beiden betroffenen Departementen - Departement für auswärtige Angelegenheiten (EDA) und Volkswirtschaftsdepartement (EVD) - zu finanzieren. Angesichts des scharfen Protests der Hilfswerke gegen Kürzungen bei der Entwicklungshilfe, der im Parlament vor allem durch die Motion Leuthard zum Ausdruck kam, sah sich der Bundesrat gezwungen, seinen Beschluss zu revidieren.

Jahrbuch 2005, Nr. 1, Kapitel 3, Debatte um EU-Kohäsionszahlungen und die Osthilfe, S. 41.

10 Swissinfo, „Kohäsion : Brüssel löst endlich den Knoten“, 8. Februar 2006.

11 Integrationbüro EDA/EVD, Mündliche Stellungnahme von Bundesrätin Micheline Calmy-Rey, Vorsteherin des Eidgenössischen Departements für auswärtige Angelegenheiten, Brüssel, 27. Februar 2006, <http://www.europa.admin.ch>. 
Die vom Parlament im Juni 2006 angenommene Motion Leuthard ${ }^{12}$ beauftragt den Bundesrat, den Beitrag der Schweiz nicht auf Kosten der öffentlichen Entwicklungshilfe zu finanzieren.

Im Juni 2006 kündigte der Bunderat eine Neuverteilung der Kompensationen des Erweiterungsbeitrags an ${ }^{13}$. 60 Prozent der Ausgaben entfallen auf die Budgets von EDA und EVD und 40 Prozent werden aus dem allgemeinen Bundeshaushalt refinanziert, insbesondere aus den Einnahmen, die sich aus der Umsetzung des mit der EU unterzeichneten Zinsbesteuerungsabkommens ergeben ${ }^{14}$. Ferner bestätigte der Bundesrat, dass die Kompensation keine Auswirkungen auf die Entwicklungshilfe für die Länder des Südens haben werde. Die Finanzierungsfrage soll erst im Sommer 2007 durch Parlamentsbeschluss geregelt werden.

\subsubsection{Nächste Etappen des Erweiterungsbeitrags}

Auf der Grundlage des neuen Gesetzes über die Ostzusammenarbeit hat der Bundesrat im Parlament die Annahme eines Rahmenkredits von einer Milliarde Franken für den Kohäsionsbeitrag der Schweiz beantragt. „Dabei handelt es sich um einen Verpflichtungskredit des Bundes über fünf Jahre. Gemäss aktuellen Berechnungen werden sich die (...) Auszahlungen über ca. zehn Jahre (20072016) erstrecken“15. Der Antrag wird von den Eidgenössischen Räten an der Frühjahrs- und Sommersession 2007 behandelt.

Nach endgültiger Annahme des Rahmenkredits erteilt das Gesetz dem Bundesrat die Kompetenz, mit den zehn Empfängerländern bilaterale Rahmenabkommen auszuhandeln. Somit wird die Schweiz - in enger Zusammenarbeit mit jedem Empfängerland - die Umsetzung ihres Beitrags zu Hilfsprojekten und -programmen festlegen. Die Verhandlungen stützen sich auf die im Memorandum of Understanding festgelegten Grundsätze. Die Verantwortung für die operationelle Projektumsetzung obliegt den Empfängerländern; die Schweiz übernimmt ihrerseits die Begleitung, Kontrolle und Evaluation der Projekte. Die ersten Projekte sollten bereits ab 2007 eingeleitet werden.

\subsection{Transitionshilfe für Osteuropa und die GUS}

Da die Gültigkeit des auf zehn Jahre befristeten Bundesbeschlusses von $1995 \mathrm{zu}$ Ende geht, musste die Gesetzesgrundlage der Transitionshilfe für Osteuropa und die GUS von den Eigenössischen Räten erneuert werden. So ermöglicht es das von Parlament und Volk angenommene Bundesgesetz über die Ostzusammenarbeit der Schweiz, ihre technische und finanzielle Zusammenarbeit mit den Oststaaten fortzusetzen.

12 05.3808, Motion Disparitätenzahlungen, eingereicht von Leuthard Doris am 14. Dezember 2005 im Nationalrat. Siehe : <http://www.parlament.ch>>Curia Vista - Geschäftsdatenbank >Suche nach Vorstössen und Geschäften.

13 EDA/EVD, „Finanzierung des Beitrags der Schweiz zur Verringerung der wirtschaftlichen und sozialen Ungleichheiten in der erweiterten EU“, Pressemitteilung vom 16. Juni 2006.

14 Das Zinsbesteuerungsabkommen sollte jährlich 50 Millionen Franken durch Zinsbesteuerung der Spareinlagen europäischer Steuerzahler in die Bundeskasse einbringen.

15 EDA/EVD, „Bundesrat genehmigt die Rahmenkredite für die traditionelle Osthilfe und den Erweiterungsbeitrag“, Pressemitteilung vom 15. Dezember 2006. 


\subsubsection{Schwerpunktbereiche und Einsatzländer von DEZA und SECO}

Die Direktion für Entwicklung und Zusammenarbeit (DEZA) und das Staatssekretariat für Wirtschaft (SECO) sind in zehn Schwerpunktländern aktiv ${ }^{16}$ und führen zwei Regionalprogramme im Südkaukasus und in Zentralasien durch. Im Jahr 2005 wurde die humanitäre Tätigkeit in Moldawien durch ein Programm für technische Zusammenarbeit ergänzt. Das Programm umfasst hauptsächlich Unterstützung in den Bereichen Mikrokredit, Berufsbildung und Verbesserung der medizinischen Behandlung.

Die den mitteleuropäischen Ländern und den Baltischen Staaten nach dem Fall der Berliner Mauer gewährte Transitionshilfe wurde ab 1997 schrittweise in andere Gebiete, vor allem nach Südosteuropa und in die GUS verlagert. Im Jahr 2004 erhielt nur noch die Slowakei Hilfe von der Schweiz in Höhe von 1,6 Millionen Franken. 2005 war die schweizerische Transitionshilfe für Mitteleuropa vollständig finalisiert.

Die zehn der Europäischen Union im Jahr 2004 beigetretenen Länder werden nach den neuen Kriterien der Organisation für wirtschaftliche Zusammenarbeit und Entwicklung (OECD) im Übrigen nicht mehr als Entwicklungshilfeempfängerländer angesehen (siehe Rahmentext).

[D] Jahrbuch 2007, Nr. 1, Statistiken, Teil C, Tabelle C.11, Bilaterale Osthilfe von DEZA und SECO, 1996-2005.

\section{Neue DAC-Liste der Hilfeempfängerländer}

2005 beschloss der Entwicklungshilfeausschuss (DAC) der OECD, die Liste der Hilfeempfängerländer zu ändern. Diese zuvor aus zwei Teilen (Empfänger öffentlicher Entwicklungshilfe und Empfänger öffentlicher Hilfe) bestehende Liste umfasst jetzt nur noch einen Teil.

Empfängerländer der öffentlichen Hilfe waren bis 2005 (gemäss Mitteilungen von 2004) folgende Staaten: Bulgarien, Estland, Lettland, Litauen, Polen, Rumänien, Russland, die Slowakei, Tschechien, die Ukraine, Ungarn und Weissrussland. Die Ukraine und Weissrussland wurden in die neue Liste der Empfängerländer öffentlicher Entwicklungshilfe integriert. Die anderen Länder werden in der DAC-Statistik nicht mehr aufgeführt.

Somit umfasst die neue Liste der Hilfeempfängerländer nur noch Empfänger der öffentlichen Entwicklungshilfe, das heisst alle Länder mit geringem und mittlerem Einkommen, ausser den Mitgliedsstaaten der G-8 oder der Europäischen Union (einschliesslich Bulgarien und Rumänien, die der EU im Januar 2007 beigetreten sind). Die neue Liste wird für Daten ab 2005 angewendet.

[D] Jahrbuch 2007, Nr. 1, DAC-Liste der Hilfeempfängerländer am Schluss des Jahrbuchs.

16 Es handelt sich um Albanien, Bosnien-Herzegowina, Bulgarien, Mazedonien, Rumänien, Serbien und die Ukraine sowie um die Russische Föderation, Moldawien und den Kosovo, für die Sonderprogramme bestehen. 
Tabelle 3.2: DEZA/SECO : Bilaterale Zusammenarbeit mit Osteuropa und der GUS nach Sektoren und Regionen, 2005 (in Millionen Franken)

\begin{tabular}{|c|c|c|c|c|}
\hline & Südosteuropa & GUS & Nicht zuteilbar & Total \\
\hline \multicolumn{5}{|l|}{ DEZA, Technische Zusammenarbeit ${ }^{a}$} \\
\hline Gouvernanz & 13.3 & 9.4 & 0.0 & 22.7 \\
\hline Gesundheit & 9.7 & 6.5 & 0.0 & 16.2 \\
\hline Umwelt, Wasser, Infrastruktur & 5.7 & 4.8 & - & 10.5 \\
\hline Landwirtschaft und ländliche Enwicklung & 3.9 & 11.0 & 0.1 & 14.9 \\
\hline Erziehung und Bildung & 6.1 & 1.2 & 0.0 & 7.4 \\
\hline Wirtschaftliche Entwicklung und Beschäftigung & 7.1 & 1.2 & - & 8.3 \\
\hline Kultur und Forschung & 5.9 & 0.5 & 0.2 & 6.7 \\
\hline Andere Sektoren & 12.3 & 3.8 & 1.4 & 17.5 \\
\hline Total DEZA 2005 & 64.0 & 38.4 & 1.8 & 104.2 \\
\hline \multicolumn{5}{|l|}{ SECO, Wirtschaftliche Zusammenarbeit } \\
\hline Infrastrukturfinanzierung & 20.5 & 18.2 & - & 38.7 \\
\hline Handelsförderung & 3.6 & 5.5 & 3.8 & 12.9 \\
\hline Investitionsförderung & 3.8 & 19.3 & 2.0 & 25.1 \\
\hline Makroökonomische Hilfe & 0.9 & 0.2 & - & 1.1 \\
\hline Andere Instrumente & 0.4 & 0.2 & 4.5 & 5.1 \\
\hline Total SECO 2005 & 29.2 & 43.4 & 10.3 & 82.9 \\
\hline Total DEZA/SECO 2005 & 93.2 & 81.8 & 12.1 & 187.1 \\
\hline Total DEZA/SECO 2004 & 98.3 & 80.5 & 24.7 & 203.6 \\
\hline
\end{tabular}

Quelle:DEZA und SECO, Jahresbericht der internationalen Zusammenarbeit der Schweiz 2005, Bern, Tabelle 10, S. 21.

a Seit 2005 haben DEZA und SECO eine neue Sektorklassifikation eingeführt. Für die DEZA waren die früheren Sektoren: Politik und Staatsaufbau, Wirtschaft/Bildung, Wissenschaft/Kultur, Landwirtschaft, Gesundheit/Soziales, Energie/Umwelt, andere Sektoren; für das SECO: Energie, Umwelt, Infrastruktur, Finanzsektor, Handel, Investitionsförderung, andere Sektoren.

\subsubsection{Folgen des Erweiterungsbeitrags für die traditionelle Hilfe}

Der Beitrag der Schweiz zur EU-Erweiterung kann Auswirkungen auf die Transitionshilfe für die Staaten Osteuropas und der GUS haben. Gemäss dem Finanzplan des Bundesrates vom Juni 2006 werden 60 Prozent der vorgesehenen Ausgaben (d.h. 60 Millionen Franken pro Jahr) durch Kürzungen des Transitionshilfebudgets kompensiert. Obwohl die genauen Folgen für die Oststaaten derzeit noch nicht vorliegen, sind insbesondere Kürzungen durch Einstellung der Hilfsprogramme für Bulgarien, Rumänien und Russland vorgesehen. Jedoch wird Brüssel von der Schweiz sicherlich einen Kohäsionsbeitrag für die Länder Bulgarien und Rumänien fordern, die der EU am 1. Januar 2007 beigetreten sind.

Das Jahresbudget der schweizerischen Hilfe für die Transitionsländer, das 200 Millionen Franken erreicht und zwischen DEZA und SECO aufgeteilt ist, wird wahrscheinlich reduziert werden. Ein neuer Jahresbetrag von 140 bis 160 Millionen Franken wurde vom Bundesrat diesbezüglich genannt ${ }^{17}$. Der neue Finanzbetrag wird jedoch vom Parlament festgelegt.

Hierzu wurde dem Parlament vom Bundesrat ein vierter Rahmenkredit für die Fortsetzung der Transitionshilfe unterbreitet. Der Bundesrat beantragte die Gewährung eines Betrags von 650 Millionen Franken für eine Laufzeit von mindestens vier Jahren ${ }^{18}$.

17 Schweizerische Eidgenossenschaft, Volksabstimmung vom 26. November 2006, Erläuterungen des Bundesrates, Informationsblatt.

18 EDA/EVD, „Bundesrat genehmigt die Rahmenkredite für die traditionelle Osthilfe und den Erweiterungsbeitrag“, Pressemitteilung vom 15. Dezember 2006. 


\section{QUELLEN}

DEZA/SECO, Jahresbericht der internationalen Zusammenarbeit der Schweiz 2005, Bern.

Integrationsbüro EDA/EVD, DEZA und SECO, „Beitrag der Schweiz zur Verringerung der wirtschaftlichen und sozialen Ungleichheiten in der erweiterten Europäischen Union", Informationsblatt, Februar 2006.

Integrationsbüro EDA/EVD, DEZA und SECO, „Bundesgesetz Ostzusammenarbeit, Transitionshilfe und Erweiterungsbeitrag“, Informationsblatt, Oktober 2006.

Rat der Europäischen Union und Schweizerischer Bundesrat, Vereinbarung, <http://www.bundesgesetzost.admin.ch > > Dokumentation >Offizielle Texte.

Alliance Sud, „Warum die Hilfswerke Ja sagen zum Osthilfegesetz“, Dossier für die Medienkonferenz vom 20. Oktober 2006, <http://www.alliancesud.ch> > Themen/Kampagnen >Entwicklungspolitik $>$ Spezialdossier EU-Kohäsionsbeitrag >Dossier Medienkonferenz vom 20. Oktober 2006.

economiesuisse, „Ohne Risiko weiter auf dem bewährten Weg. Konstruktive Zusammenarbeit mit Osteuropa“, dossierpolitik Nr. 35.2, 9. Oktober 2006.

SVP, „Bezahlen ohne Ende? 2xNein zum Zulagen-Gesetz und den Ost-Milliarden“, Argumentarium, 25. September 2006.

Le Temps, „Une histoire de milliard“, 30. Oktober 2006.

\section{INTERNET-ADRESSEN}

Bundesversammlung, Zusammenarbeit mit Osteuropa : <http://www.parlament.ch/d/do-osthilfe > .

EDA und EVD, Beitrag zur Erweiterung der Europäischen Union : < http://www.erweiterungsbeitrag.ch>. Integrationsbüro EDA/EVD: <http://www.europa.admin.ch>. 